TRANSACTIONS OF THE

AMERICAN MATHEMATICAL SOCIETY

Volume 349, Number 10, October 1997, Pages 3925-3943

S 0002-9947(97)01921-1

\title{
TWO DECOMPOSITIONS IN TOPOLOGICAL COMBINATORICS WITH APPLICATIONS TO MATROID COMPLEXES
}

\author{
MANOJ K. CHARI
}

\begin{abstract}
This paper introduces two new decomposition techniques which are related to the classical notion of shellability of simplicial complexes, and uses the existence of these decompositions to deduce certain numerical properties for an associated enumerative invariant. First, we introduce the notion of $M$-shellability, which is a generalization to pure posets of the property of shellability of simplicial complexes, and derive inequalities that the rank-numbers of M-shellable posets must satisfy. We also introduce a decomposition property for simplicial complexes called a convex ear-decomposition, and, using results of Kalai and Stanley on $h$-vectors of simplicial polytopes, we show that $h$-vectors of pure rank- $d$ simplicial complexes that have this property satisfy $h_{0} \leq h_{1} \leq \cdots \leq h_{[d / 2]}$ and $h_{i} \leq h_{d-i}$ for $0 \leq i \leq[d / 2]$. We then show that the abstract simplicial complex formed by the collection of independent sets of a matroid (or matroid complex) admits a special type of convex eardecomposition called a $P S$ ear-decomposition. This enables us to construct an associated M-shellable poset, whose set of rank-numbers is the $h$-vector of the matroid complex. This results in a combinatorial proof of a conjecture of Hibi [17] that the $h$-vector of a matroid complex satisfies the above two sets of inequalities.
\end{abstract}

\section{INTRODUCTION}

This paper addresses a fundamental invariant associated with the abstract simplicial complex (henceforth called a matroid complex) defined by the collection of independent sets of a matroid. The motivation for this work and the primary results are essentially combinatorial in nature, however the techniques that are used have a distinctly topological flavor. In particular, we will study the geometric realization of the matroid complex and show that it satisfies a certain topological decomposition property which eventually leads to the combinatorial results about this invariant. To describe the problem and nature of the techniques and results in greater detail, we will need some elementary definitions and terminology.

For a finite, abstract, pure, rank- $d$ simplicial complex $\Sigma$, the $i$-faces of $\Sigma$ are the elements of $\Sigma$ of cardinality $i$. We will refer to the $d$-faces as facets of $\Sigma$. The $f$-vector of $\Sigma$ is the integer vector $\left(f_{0}, f_{1}, \ldots, f_{d}\right)$, where $f_{i}$ is the number of its $i$-faces. The $f$-vector is the enumerative invariant of a simplicial complex that comes to mind first, however one can also define a related invariant called the $h$-vector as the vector $\left(h_{0}, h_{1}, \ldots, h_{d}\right)$, where $h_{i}$ is the coefficient of $x^{d-i}$ in the

Received by the editors May 6, 1995.

1991 Mathematics Subject Classification. Primary 52B40; Secondary 06A07.

Key words and phrases. Matroid complex, shellability, simplicial complex, boundary complex, simplicial polytope, $h$-vector.

(C)1997 American Mathematical Society 
polynomial $\sum_{i=0}^{d} f_{i}(x-1)^{d-i}$. The seminal work of McMullen [21],[22] and Stanley ([26], [27], [28], etc.) has shown that the $h$-vector encapsulates crucial algebraic, enumerative and topological properties of simplicial complexes that are shellable. The notion of shellability of boundary complexes of convex polytopes and, more generally, cell complexes has a long history [34]. However, we will restrict ourselves to the framework of abstract simplicial complexes, where this topological notion has the following equivalent and purely combinatorial formulation.

A pure rank- $d$ simplicial complex is shellable if there exists an ordering (called a shelling) $F_{1}, F_{2}, \ldots, F_{m}$ of its facets with the following property:

For every $1 \leq i<j \leq m$, there exists $h \leq j$ such that $F_{i} \cap F_{j} \subseteq F_{h} \cap F_{j}$ and $\left|F_{h} \cap F_{j}\right|=d-1$.

Several important classes of simplicial complexes that arise in combinatorial mathematics, such as matroid complexes, boundary complexes of simplicial polytopes, broken circuit complexes [9], are known to be shellable ([8],[25],[14]). Using algebraic methods, Stanley [26] showed that the $h$-vector of any shellable simplicial complex is the degree sequence of some order ideal of monomials (in short, an O-sequence). An explicit numerical characterization for O-sequences is well known due to Macaulay [26], and this gives a set of numerical restrictions on the set of the $h$-vectors of shellable complexes. (The proper framework for these results is the theory of Cohen-Macaulay complexes developed by Baclawski, Hochster, Reisner and Stanley, and we refer to [28] for details). Since then research has focussed on obtaining separate characterizations or at least stronger restrictions for $h$-vectors for each of these important classes of complexes. A singular accomplishment in this area of mathematics was the complete resolution (c. 1980) of McMullen's conjectured combinatorial characterization of the set of $h$-vectors of simplicial polytopes. Stanley's proof of the necessity of McMullen's conditions involved the algebraic geometry of toric varieties, while the subsequent sufficiency proof of Billera and Lee was combinatorial [27], [28]. An alternative necessity proof using polytope algebras has recently been given by McMullen [23].

In comparison, progress on the fundamental problem of characterizing the $h$ vectors of matroid complexes has been extremely limited. Several unimodality and log-concavity conjectures have been made about the $f$-vectors and $h$-vectors of matroid complexes [4], [31], [11], though proofs are known only for very special cases. We believe that the results of this paper represent tangible progress towards the goal of identifying a plausible characterization for $h$-vectors of matroid complexes. The research in this paper was primarily motivated by the following conjecture of Stanley [26], and a weaker version thereof, formulated more recently by Hibi [17]. (Note: A pure O-sequence is the degree sequence of an order ideal of monomials, whose maximal elements are all of the same degree.)

Conjecture 1 (Stanley). The h-vector of a matroid complex is a pure O-sequence.

A complete numerical characterization is not known for pure O-sequences, and indeed this problem is widely regarded to be extremely difficult, if not intractable. (See the discussion in the last section.) However, Hibi [16] has shown that a pure O-sequence $\left(h_{0}, h_{1}, \cdots, h_{d}\right)$ must satisfy the following two conditions:

$$
\begin{gathered}
h_{0} \leq h_{1} \leq \cdots \leq h_{[d / 2]} \\
h_{i} \leq h_{d-i}, \quad 0 \leq i \leq[d / 2] .
\end{gathered}
$$


((1) is often referred to as the generalized lower bound conjecture (GLBC) in the context of $h$-vectors of simplicial spheres [30].)

This result led Hibi [17] to formulate the following weaker version of Stanley's conjecture.

Conjecture 2 (Hibi). The h-vector of a matroid complex must satisfy inequalities (1) and (2).

In that paper, Hibi [17] established the following partial result in the direction of $(2)$ :

$$
h_{0}+h_{1}+\ldots+h_{i} \leq h_{d}+h_{d-1}+\cdots+h_{d-i}
$$

for $i \leq[d / 2]$.

The following recent result of Brown and Colbourn [7] is perhaps more interesting, since it shows that Stanley's conjectured necessary condition for $h$-vectors of matroid complexes is certainly not sufficient-for example, consider the pure O-sequence $(1,4,2)$ !

Proposition 1 (Brown and Colbourn). The h-vector of a connected rank-d matroid satisfies the following:

$$
(-1)^{j} \sum_{i=0}^{j}(-b)^{i} h_{i} \geq 0, \quad 0 \leq j \leq d,
$$

for any positive real number $b \geq 1$, with equality possible only if $b=1$.

The two results that we just mentioned were, to the best of our knowledge, the strongest general restrictions known in the literature for $h$-vectors of matroids. Our decomposition techniques will enable us to prove (1), (2), and (3) for $h$-vectors of matroid complexes as a corollary of a topological decomposition theorem for matroids along with some purely poset-theoretic results of Section 2 . Though we are unable to prove Stanley's conjecture in this paper, our results suggest a stronger condition for $h$-vectors of matroids, which we present at the end of the paper as a conjecture.

The techniques introduced in this paper derive their initial inspiration from Björner's work [4] (which appeared first in a 1979 preprint, see also [12]), in which for any given matroid complex, he gives an explicit combinatorial description of a set of spherical subcomplexes of the matroid complex which are joins of simplicial boundaries. The primary result of Björner is that the fundamental cycles of these subcomplexes form a basis for the only non-trivial homology group of the matroid complex and, further, the original matroid complex is the union of these subcomplexes. We take a completely different approach by developing a recursive algorithm to construct a concrete topological decomposition for matroid complexes. The resulting "pieces" of the matroid complex, however, have essentially the same structure as Björner's subcomplexes. The nature of the decomposition reveals an interesting relation between the structure of matroid complexes and boundary complexes of simplicial polytopes and their subcomplexes which, to the best of our knowledge, has not been explored in the literature.

This paper assumes familiarity with basic concepts of matroid theory [32], [24], the theory of posets [29], and the elements of combinatorial topology [6]. Other references which discuss the background directly related to this paper in detail 
include Stanley [28], Billera [2] Björner [4], and Colbourn [11]. In Section 2, we introduce a set of structural decomposition properties called $k$-decomposability, $M$ partitionability and $M$-shellability for pure posets, which are generalizations of the properties of $k$-decomposability [25], interval-partitionability and shellability for simplicial complexes. The primary enumerative result in this theory is that the existence of the weakest decomposition of these properties, namely M-partitionability, for a pure poset implies that the set of rank numbers of the poset must satisfy the inequalities (1), (2), and (3).

In Section 3, we discuss a decomposition, called a convex ear-decomposition, associated with simplicial complexes, which can be interpreted as a higher-dimensional analog of the notion of ear-decomposition of graphs [19]. Using results of Kalai and Stanley, we show that the $h$-vectors of simplicial complexes that admit such a decomposition satisfy inequalities (1) and (2). We then show that complexes that admit a special type of convex ear-decomposition called a PS ear-decomposition admit a shelling, whose lower-bound poset is M-shellable in the sense of Section 2. This poset is defined so that its set of rank numbers equals the $h$-vector of the simplicial complex. In particular, we establish that matroid complexes have this property, thus providing a strictly combinatorial proof that $h$-vectors of matroid complexes satisfy inequalities (1),(2), and (3) - thus proving Hibi's conjecture.

\section{A POSET-Theoretic GenERALIZATION OF SHELlability}

2.1. Preliminaries and definitions. We will assume familiarity with posets and refer to Stanley's book [29] for terminology and elementary properties of posets and poset operations. In what follows, we will assume that all posets are finite. We will denote by $C(P)$ the set of cover relations of $P$. A poset $P$ is a ranked poset, if it has a unique minimal element denoted by $\hat{0}$ and, further, for any element $x$ of $P$ every saturated chain from $\hat{0}$ to $x$ of the poset has the same length, which is defined to be its rank and is denoted by $\rho(x)$. A poset $P$ is pure rank-d if it is a ranked poset and all its maximal elements are of rank $d$.

We say that $Q$ is an edge-induced subposet of $P$ if $Q \subseteq P$ as sets and $C(Q)=$ $\{(x, y): x, y \in Q,(x, y) \in C(P)\}$. We should point out that this concept is somewhat different from the traditional notion of induced subposet, where the partial order itself is induced on to the subset of elements and not just the cover relations as in our case. The rationale for our particular choice will be clear later in the paper.

For an element $x$ of a poset $P$, let $\mathcal{F}(x)$ represent the order filter generated by $x$, that is, $\mathcal{F}(x)$ is the edge-induced subposet on $\{z \in P: x \leq z\}$. Also, we define the poset $\Gamma(x)$ as the edge-induced subposet on the set $P-\mathcal{F}(x)$.

Definition. A poset $Q$ is an $M$-poset if there exists a monomial $\mathrm{M}$ on a finite set $E$ of indeterminates such that $Q$ is isomorphic to the poset (ordered by divisibility) on the set of monomials on $E$ that divide $\mathrm{M}$.

Equivalently, an M-poset is a direct product of chains. Hence, for $d \geq 0$, the simplest example of such a rank- $d$ M-poset is a $d$-chain.

Given two elements $x \leq y$ of $P$, the interval $[x, y]$ is called an $M$-interval if it is an M-poset.

Definition. A pure poset $P$ is $M$-partitionable if $P$ can be partitioned into Mintervals $\left[x_{i}, y_{i}\right], i=1,2, \ldots, n$, such that each $y_{i}$ is a maximal element of the poset $P$. Such a partition is called an $M$-partition of the poset $P$. 
Definition. An $M$-shelling of a poset $P$ is an M-partition of $P$ along with an ordering of the M-intervals such that the union of the elements in any initial subsequence of M-intervals in the ordering of intervals is an order ideal of $P$. A poset $P$ is $M$-shellable, if it admits an M-shelling.

We leave it to the reader to construct an example to show that M-shellability is a strictly stronger property. We will refer to the elements $x_{i}$ and $y_{i}$ associated with an M-interval as the lower bound and upper bound associated with the M-interval.

If $P$ is a simplicial complex, then the above definitions reduce to the classical definitions of partitionability and shellability. (See the first lemma of Section 2.4.) We would have liked to use the terms partitionable and shellable posets, however this expression has been used extensively (see [3], [4], [29]) for pure posets, whose chain complexes satisfy the classical notions of shellability and interval-partitionability.

Now we define an important poset associated with an M-shelling of a poset.

Definition. Given an M-shelling $S$ of a pure rank- $d$ poset of $P$, we define the lower-bound poset associated with $S$, denoted by $\Delta(S, P)$, to be the edge-induced subposet of $P$ on the set of lower bounds of $S$.

Next, we define a hierarchy of combinatorial decomposition properties which are generalizations of the theory of $k$-decomposability of simplicial complexes developed by Provan and Billera ([25]).

Definition. Let $P$ be a pure rank- $d$ poset. For an integer $k, 1 \leq k, P$ is $k$ decomposable if $P$ is an M-poset, or there exists an element $x$ of $P$, with $\rho(x) \leq k$, (called a k-element of $\mathrm{P}$ ) such that

1. $\mathcal{F}(x)$ is a pure rank- $(d-\rho(x))$ poset and is $k$-decomposable, and

2. $\Gamma(x)$ is a pure rank- $d$ poset and is $k$-decomposable.

Clearly $k$-decomposability of posets forms a heirarchy of properties, that is, $k$ decomposability implies $k+1$-decomposability. Note that $k$-decomposability for $k>d$, where $d$ is the rank of the poset, is equivalent to $d$-decomposability. Thus in (1) of the above definition, we could equivalently require $k_{1}$-decomposability of $\mathcal{F}(x)$ where $k_{1}=\min \{k, d-\rho(x)\}$. Also, it is easy to see that the requirement of purity of $\mathcal{F}(x)$ in (1) is redundant, but it is included to make the definition more symmetric. When $k=1$, we will call the property atom-decomposability, which generalizes the property of vertex-decomposability of simplicial complexes defined by Provan and Billera. It is easy to show that many interesting classes of complexes, including matroid complexes and broken-circuit complexes, have this property [25]. At the present time, we do not have any new examples of interesting classes of $k$-decomposable posets for $k<d$. Indeed, our primary interest in this paper is in the property of M-shellability, which we will relate in the next section to the notion of $k$-decomposability. We have developed a more general approach through $k$-decomposability to emphasize the fact that M-shellability has an equivalent recursive formulation (see (2) of Theorem 1), which is certainly not obvious from its original definition. We also hope that other interesting applications of this more general decomposition theory can be found in the future.

2.2. Some structural results. Many aspects of the $k$-decomposability theory for simplicial complexes of [25] can be generalized to our framework, however we will state and prove only the most significant of these properties in the next theorem. When more than one poset is involved, we will use subscripts or brackets to indicate the poset with respect to which a certain function or subposet is defined. We will 
assume familiarity with the notion of the direct product of two posets $P$ and $Q$, which we denote by $P \times Q$. We now define two other useful poset operations.

1. The $i$-truncation of a pure rank- $d$ poset $P$, where $0 \leq i \leq d$, is the pure rank $i$-poset $P^{i}=\{x \in P: \rho(x) \leq i\}$.

2. An $i$-join of a pure rank- $d$ poset $P$ with respect to a pure rank- $(d-i)$ poset $Q$ is defined only if $1 \leq i \leq d$, and the two posets are (set-wise) disjoint. Such a poset is denoted by $P_{i} \nearrow Q$, and its Hasse diagram is obtained taking the "disjoint union" of the Hasse digrams of the two posets and making the $\hat{0}(Q)$ cover some element of $P$ of rank $i-1$, and then letting some (possibly none) of the elements of $Q$ cover the elements of $P$ of the appropriate rank so that the resulting poset is a pure rank- $d$ poset. (Strictly speaking, when we talk of an $i$-join we must specify the new cover relations that are introduced, but for our purposes this will not be relevant and for brevity's sake we shall use the generic term $i$-join.)

\section{Theorem 1.}

1. The direct product of $k$-decomposable posets is $k$-decomposable.

2. A pure rank-d poset is d-decomposable if and only if it is M-shellable.

3. The direct product of $M$-partitionable posets is also M-partitionable. Given $M$-shellings $S_{1}$ and $S_{2}$ of two posets $P$ and $Q$ which are pure rank- $d_{1}$ and pure rank- $d_{2}$ respectively, there exists on $M$-shelling $S$ of the direct product $P \times Q$ such that $\Delta(S,(P \times Q))=\Delta\left(S_{1}, P\right) \times \Delta\left(S_{2}, Q\right)$.

4. For $1 \leq i \leq d$, the $i$-truncation of an $M$-partitionable (M-shellable) rank-d poset $P$ is a rank-i M-partitionable (M-shellable) poset.

5. For $1 \leq i \leq d$, an $i$-join of an $M$-shellable (M-partitionable) rank-d poset $P$ with an $M$-shellable (M-partitionable) poset rank- $(d-i)$ poset $Q$ is a rank-d M-shellable (M-partitionable) poset.

Proof. 1. Suppose $P$ and $Q$ are pure $k$-decomposable posets of rank $d_{1}$ and $d_{2}$ respectively. Clearly $P \times Q$ is an M-poset if and only if both $P$ and $Q$ are, and $P \times Q$ is pure if and only if both $P$ and $Q$ are. Now suppose $P \times Q$ is not an M-poset, then we can assume without loss of generality that $P$ is not an M-poset. Let $x_{p}$ be a $k$-element of $P$. Now observe that $\Gamma_{P \times Q}\left(x_{p}, \hat{0}(Q)\right)=\Gamma_{P}(x) \times Q$, while $\mathcal{F}_{P \times Q}\left(x_{P}, \hat{0}(Q)\right)=\mathcal{F}_{P}(x) \times Q$. It follows by induction and the $k$-decomposability of $P$ and $Q$, that $\left(x_{p}, \hat{0}(Q)\right)$ is a $k$-element for $P \times Q$.

2. First, we show, using induction on the number of elements of $P$, that a pure rank- $d d$-decomposable poset $P$ is M-shellable. Clearly the result is true, when $|P|=d+1$. $(d+1$ is the smallest number of elements $P$ can have. $)$ Now the result is also true if $P$ is an M-poset. Suppose $P$ is not an M-poset, then there exists a $d$-element $x$ such that $\Gamma_{P}(x)$ and $\mathcal{F}_{P}(x)$ are pure rank- $d$ and pure rank$\left(d-\rho_{P}(x)\right)$ posets respectively and are both $d$-decomposable. Note that for $\mathcal{F}_{P}(x)$, the $d$-element (which exists unless it is an M-poset) actually has rank at most $\left(d-\rho_{P}(x)\right)$, and hence $\mathcal{F}_{P}(x)$ is actually a $\left(d-\rho_{P}(x)\right)$-decomposable poset. Thus it follows by induction that both $\Gamma_{P}(x)$ and $\mathcal{F}_{P}(x)$ are M-shellable, and now it is easy to construct an M-shelling of $P$ from those two M-shellings.

Now to prove the converse, let $P$ be a rank- $d$ M-shellable poset and let $x$ be the lower bound of the last M-interval of $P$ in some M-shelling of $P$. Of course, if $x=\hat{0}(P)$ then $P$ is an M-poset and we are done. Otherwise, we claim that $x$ is a $d$-element for $P$. Clearly, we have that $\mathcal{F}(x)$ is a rank- $(d-\rho(x))$ M-poset. Now, 
evidently $\Gamma_{P}(x)$ is a pure rank- $d \mathrm{M}$-shellable poset, and hence, by induction, it is $d$-decomposable. It follows that $P$ is $d$-decomposable.

3. The proofs of these two statements follow from the following simple observations. First, the direct product of two M-posets is an M-poset, and further, the direct product can be distributed across a disjoint union of subposets. Also a subposet is an order ideal in $P \times Q$ if it is the product of order ideals of $P$ and $Q$. The proof of (3) is now straightforward.

4. Observe that the $(d-1)$-truncation of a rank- $d$ M-poset is M-shellable. It is now straightforward to construct an M-shelling of the $(d-1)$-truncation of $P$ from the M-shellings of the truncation of each M-interval. The above procedure can be applied repeatedly to get the result for general $i$.

5. We omit the easy proof of the result for $i$-joins. This completes the proof of the theorem.

2.3. Rank-number inequalities. Clearly, M-partitionability and M-shellability imply a very specific structure for the poset. Indeed, we can derive interesting enumerative consequences for posets that admit such special structure.

Let $F(t)=\sum_{i=0}^{d} f_{i} t^{i}$ be the rank generating function of the poset $\mathrm{P}$, so that $f_{i}$ is the number of rank- $i$ elements of $P$. Now let the $i$ th M-interval $\left[x_{i}, y_{i}\right]$ in the M-partition of a given M-partitionable pure rank- $d$ poset be isomorphic to the ideal generated by the monomial $M_{i}=z_{1}^{r_{i, 1}} z_{2}^{r_{i, 2}} \ldots z_{s_{i}}^{r_{i, s_{i}}}$. Hence, we have $d=$ $\rho\left(x_{i}\right)+r_{i, 1}+\ldots+r_{i, s_{i}}$ for every $i=1,2, \ldots, n$. Now, let us define for any nonnegative integer $k$, a polynomial $u(k ; t)$ as follows:

$$
u(k ; t)=t^{0}+t^{1}+\cdots+t^{k} .
$$

Thus, $u(k ; t)$ is the rank generating function of the $k$-chain.

Proposition 2. The rank generating function $F(t)$ of an $M$-partitionable poset satisfies the following equation:

$$
F(t)=\sum_{i=1}^{n} t^{\rho\left(x_{i}\right)} u\left(r_{i, 1} ; t\right) u\left(r_{i, 2} ; t\right) \cdots u\left(r_{i, s_{i}} ; t\right)
$$

Proof. It is easy to verify that each term in the sum corresponds exactly to the sum of the individual terms for the elements in a fixed M-interval. The product form follows from the fact that each M-interval is an M-poset.

Proposition 3. The rank numbers $\left(f_{0}, f_{1}, f_{2}, \ldots, f_{d}\right)$ of a rank-d M-partitionable poset $P$ satisfy the following sets of inequalities:

$$
\begin{gathered}
f_{0} \leq f_{1} \leq \cdots \leq f_{[d / 2]}, \\
f_{i} \leq f_{d-i}, \quad 0 \leq i \leq[d / 2], \\
(-1)^{j} \sum_{i=0}^{j} f_{i}(-b)^{i} \geq 0, \quad 1 \leq j \leq d,
\end{gathered}
$$

for any positive real number $b \geq 1$, with equality possible only if $b=1$. 
Proof. Consider any element $a$ in the poset $P$ with $\rho(a) \leq[d / 2]$. Let $\left[x_{j}, y_{j}\right]$ be the unique M-interval of the M-partition to which $a$ belongs. It is well-known ([16], [1]) that any poset which is a direct product of chains admits a symmetric saturated chain decomposition. Thus $a$ belongs to a unique saturated chain $\mathrm{C}$ which is contained in $\left[x_{j}, y_{j}\right]$ and symmetric with respect to this interval. Since $\rho\left(x_{j}\right) \leq \rho(a) \leq[d / 2]$ and $\rho\left(y_{j}\right)=d$, the rank of the top element of $\mathrm{C}$ is at least $d-\rho(a)$. Thus we can define maps $\phi_{j}(a)$ for $[d / 2] \geq j \geq \rho(a)$ and for $j=d-\rho(a)$, such that $\phi_{j}(a)$ in an element of the chain $\mathrm{C}$ and $\rho\left(\phi_{j}\right)=j$. It is easy to see that each such map $\phi_{j}$ is an injective map from the set of elements of rank $\rho(a)$ to the set of elements of rank $j$. The first two inequalities follow from the existence of these injections.

We will use the rank generating function to prove the third inequality, noting that we could also prove the first two inequalities using the expansion of $F(t)$. Since $j$-truncations of M-partitionable posets ((4) of Theorem 1) are M-partitionable, it suffices to prove the inequality for $j=d$.

We address the case $b=1$ first. Note that if $t=-1$ in the product

$$
u\left(r_{i, 1} ; t\right) u\left(r_{i, 2} ; t\right) \cdots u\left(r_{i, s_{i}} ; t\right),
$$

we obtain 1 exactly when the highest exponent in each polynomial is even, while for any other instance, the product is zero. So if the product is 1 , we must have that $d-\rho\left(x_{i}\right)$ (which is the sum of the individual highest exponents) is even. Hence, $(-1)^{-d} F(-1) \geq 0$, which is equivalent to inequality for $b=1$.

Now if $b>1$, we have $u(k ;(-b))=\left((-b)^{k+1}-1\right) /(-b-1)$, and therefore $(-1)^{k} u(k ;(-b))>0$ for all non-negative integers $k$. The strict inequality follows quite easily now from the expansion of $F(t)$.

The proof of the proposition is now complete.

2.4. The lower-bound poset of a shelling. The following classical result of McMullen [21] indicates that the definitions of M-shellability and M-partitionability given in the previous sections reduce to the classical definition of shellability and interval-partitionability when $P$ is an abstract pure rank- $d$ simplicial complex, ordered by inclusion.

Lemma 1. For a pure simplicial complex $\Sigma$, and a given ordering $F_{1}, F_{2}, \ldots, F_{m}$, the following are equivalent:

1. $F_{1}, F_{2}, \ldots, F_{m}$ is a shelling.

2. There exist sets $G_{i} \subseteq F_{i}, i=1,2, \ldots, m$, such that $\Theta=\left\{\left[G_{i}, F_{i}\right], i=\right.$ $1,2, \ldots, m\}$ is an interval-partition of $\Sigma$ and, further, $\bigcup_{i=1}^{j}\left[G_{i}, F_{i}\right]$ is a simplicial complex for $j=1,2, \ldots, m$.

Further, if $\left(h_{0}, h_{1}, \ldots, h_{d}\right)$ is the $h$-vector of $\Sigma$, then we have

$$
h_{i}=\left|\left\{G_{j}:\left|G_{j}\right|=i, 1 \leq j \leq m\right\}\right| .
$$

In particular, this means that the number of lower bounds of a certain cardinality is independent of the particular shelling. Henceforth, we will use the classical terminology when dealing with complexes and use the terms M-intervals, Mpartitionable, etc. only when dealing with posets which are not complexes. Henceforth, we will abuse notation and also use the term shelling to denote the ordered sequence of intervals in the interval-partition. 
The rationale behind using the edge-induced subposet is clear from the following lemma, which appears as Lemma 7.2.6 in [4] but we will include a proof for the sake of completeness.

Lemma 2. Let $\Sigma$ be a simplicial complex and $S$ be a shelling for $\Sigma$. Then $\Delta(S, \Sigma)$ is a poset with a well-defined rank function, and the set of rank-numbers of $\Delta(S, \Sigma)$ is equal to the h-vector of $\Sigma$.

Proof. Obviously, $\emptyset$ is an element of $\Delta(S, \Sigma)$. We will show that for any nonempty lower bound $F$ associated with the shelling $S$, there exists a saturated chain of length $|F|$ in $\Delta(S, \Sigma)$ from $\emptyset$ to $F$. For this, it suffices to show that for some element $x$ in $F, F-\{x\}$ is also a lower bound of $S$.

Now let $[L, U]$ be the last interval (clearly, well-defined) of the shelling $S$ whose lower bound is a proper subset of $F$. We claim that $L=F-\{x\}$ for some $x \in F \backslash U$. To show this, let $x$ be any element of $F-L$. By definition of $[L, U]$, it is clear that $F-\{x\}$ must be an element of $[L, U]$ and hence $F-\{x\} \subseteq U$. So if $x$ and $y$ are two distinct elements of $F-L$, then we have $F-\{x\} \subseteq U$ and $F-\{y\} \subseteq U$, implying that $F \subseteq U$, which is impossible. Therefore, $F-L$ is a singleton set as required.

Thus $\emptyset$ is indeed the unique minimal element of $\Sigma$. From the fact that $\Delta(S, \Sigma)$ is an edge-induced subposet of $\Sigma$, it is evident that any chain from the minimal element to $F$ also exists in $\Sigma$, and hence is of the same length, that is, $|F|$.

Corollary 1. If the lower-bound poset of some shelling of a rank-d simplicial complex $\Sigma$ is a pure rank-d M-partitionable poset, then the $h$-vector of the simplicial complex satisfies inequalities (1), (2) and (3).

Example 1. Consider the simplicial complex $\Sigma$ whose facets are 13, 14, 23, 24. Observe that this particular order $S$ is a shelling, and $\Delta(S, \Sigma)$ is an M-poset with elements $\emptyset, 4,2$, and 24 . However, for the shelling $S_{1}$ given by the order $13,23,24,14$, $\Delta\left(S_{1}, \Sigma\right)$ has elements $\emptyset, 2,4,14$, and hence is not even pure! Thus, lower-bound posets associated with different shellings of the same simplicial complex can have completely different structural properties, though their set of rank-numbers is an invariant of the simplicial complex.

Example 2. If $\Sigma$ is the collection of all proper subsets of a 4 -element set, then it is evident that any ordering of its facets gives rise to a shelling, and the lower-bound poset for any such shelling is a 3-chain. In general, one can easily show that for every shelling of the boundary of a $d$-simplex, the resulting lower-bound poset is a $d$-chain. Further, from (3) of Theorem 1, direct products of such complexes admit a shelling such that the lower-bound poset is an M-poset of rank equal to the sum of the ranks of the individual complexes.

The above results and examples clearly suggest that to be able to infer anything useful about the $h$-vector from the lower-bound poset, it is important to be able to construct shellings, so that the associated posets are "well-structured".

\section{A topologicAl DECOMPOSITION}

3.1. Preliminaries. For the purpose of this section, we will assume familiarity with the combinatorial theory of polytopes and polyhedra [34] and also with some basic concepts of combinatorial topology to the extent of [6]. In the context of simplicial complexes, direct product corresponds to the notion of join, but for the 
sake of consistency with the previous section, we will continue to use the posettheoretic term. We will also continue to use the notion of rank when talking about general simplicial complexes, but we will also use the notion of dimension whenever the simplicial complex triangulates a $d$-sphere or a $d$-ball, in which case we will call the complex a $d$-sphere or a $d$-ball. Recall that the boundary of a $(d-1)$-ball $\Sigma$, which we denote by $\partial \Sigma$, is the pure rank- $(d-1)$ simplicial complex (which is a $(d-2)$-sphere) whose facets are the rank- $(d-1)$ faces of $\Sigma$ that are contained in only one facet of $\Sigma$. We will denote the $((|X|-1)$-dimensional $)$ simplex on the set $X$ by $\bar{X}$, and denote its boundary by $\partial \bar{X}$. The boundary complex of a simplicial polytope is the simplicial complex obtained by representing each "geometric" face of the simplicial polytope by the set of its vertices.

Given a nonempty face $F$ of a simplicial complex $\Sigma$, we define

$$
\operatorname{link}(\Sigma, F)=\{G \in \Sigma:(G \cup F) \in \Sigma,(G \cap F)=\emptyset\} .
$$

We define $\Sigma \backslash F$ as the simplicial complex $\{G \in \Sigma: F$ is not a face of $G\}$.

3.2. Convex ear-decompositions and $h$-vectors. We begin with the following important definition.

Definition. A convex ear-decomposition of a pure rank- $d$ simplicial complex $\Sigma$ is an ordered sequence $\Sigma_{1}, \Sigma_{2}, \ldots, \Sigma_{m}$ of pure rank- $d$ subcomplexes of $\Sigma$ such that

1. $\Sigma_{1}$ is the boundary complex of a simplicial $d$-polytope, while for each $i=$ $2, \ldots, m, \Sigma_{i}$ is a $(d-1)$-ball which is a (proper) sub-complex of the boundary complex of a simplicial $d$-polytope, and

2. For $i \geq 2, \Sigma_{i} \cap\left(\bigcup_{j=1}^{i-1} \Sigma_{j}\right)=\partial \Sigma_{i}$.

Let $h(\Gamma)$ represent the $h$-vector of the rank- $d$ simplicial complex $\Gamma$ and let $h^{r}(\Gamma)$ represent the $h$-vector of $\Gamma$ in reverse order. Thus if $h(\Gamma)=(1,2,0)$ for a rank-2 simplicial complex $\Gamma$, then $h^{r}(\Gamma)=(0,2,1)$.

Lemma 3. If $\Sigma_{1}, \Sigma_{2}, \ldots, \Sigma_{m}$ is a convex ear-decomposition of $\Sigma$, then we have the following:

$$
h(\Sigma)=h\left(\Sigma_{1}\right)+\sum_{i=2}^{m} h^{r}\left(\Sigma_{i}\right)
$$

Proof. It is well-known (see the proof of Lemma 2.3 in [30]) that if $\Omega$ triangulates a $(d-1)$-ball, then

$$
\sum_{F \in \Omega-\partial \Omega} f_{i}(x-1)^{d-|F|}=\sum_{i=0}^{d} h_{i} x^{i},
$$

where $\left(h_{0}, h_{1}, \ldots, h_{d}\right)$ is the $h$-vector of $\Omega$. The proof of the lemma follows immediately.

We now state a recent result, originally due to Kalai [18], which is proved using a more general algebraic framework by Stanley [30].

Proposition 4 (Kalai, Stanley). The h-vector of a $(d-1)$-ball which is a subcomplex of a simplicial d-polytope satisfies the following:

$$
h_{d} \geq h_{d-1} \geq \cdots \geq h_{[d / 2]},
$$




$$
h_{i} \geq h_{d-i}, \text { for } 0 \leq i \leq[d / 2] .
$$

Corollary 2. Let $\Sigma$ be a pure rank-d simplicial complex which admits a convex ear-decomposition. Then the h-vector of $\Sigma$ satisfies inequalities (1) and (2).

Proof. The $h$-vector of $\Sigma_{1}$ is symmetric and also unimodal due to Stanley's result [27] on the necessity of McMullen's conditions for boundary complexes of simplicial polytopes. The proof of the corollary now follows immediately from Lemma 3 and Proposition 4.

The symmetry of the $h$-vector (also called the Dehn-Sommerville equations) is known for arbitrary triangulations of spheres, however the question of whether the GLBC inequality $h_{0} \leq h_{1} \leq \cdots \leq h_{[d / 2]}$ holds for all triangulations of spheres or even shellable triangulations is not yet settled. We could have defined a more general notion of, say, spherical ear-decompositions, by only requiring the first subcomplex to be a $(d-1)$-sphere and rest of the complexes to be $(d-1)$-balls. However, this generalization does not seem worthwhile at this time, since the above corollary cannot be extended to such a framework.

3.3. PS ear-decompositions and M-shellability. We now define a very specific type of convex ear-decomposition of a simplicial complex, which we call a $P S$ ear-decomposition. ("PS" represents the fact that products of simplices and their boundaries are involved in a crucial way.) For complexes that admit such an eardecomposition, an alternative, purely combinatorial proof of the inequalities (1) and (2) for the $h$-vector can be given. This is because the PS ear-decomposition naturally leads to a shelling of a matroid complex such that the lower bounds poset of the shelling is a pure, M-shellable poset.

Definition. A $P S-d$-sphere is a $d$-sphere which is the direct product of boundaries of simplices. (The complex consisting of only the empty set is a PS-0-sphere.) A $P S$ - $d$-ball is a $d$-ball which is the direct product of a simplex and a PS-sphere.

It is easy to verify the following from the above definitions:

(i) A PS- $(d-1)$-sphere is the boundary complex of some simplicial $d$-polytope.

(ii) A PS-ball is a simplicial complex of the form $\Sigma \backslash v$, where $v$ is some vertex (rank-1 face) of a PS-sphere.

(iii) The boundary of PS- $d$-ball is a PS- $(d-1)$-sphere.

(iv) The direct product of PS-spheres is a PS-sphere, while the direct product of a PS-ball with a PS-sphere is a PS-ball.

Remark. It can be shown that a matroid complex triangulates a sphere ([4], page 278 ) if and only if each connnected component of the matroid is a circuit. Since the matroid complex of a circuit is the boundary of a simplex, we have that if a rank- $d$ matroid complex triangulates a sphere then it is a PS- $(d-1)$-sphere.

Definition. A $P S$ ear-decomposition of a pure rank- $d$ simplicial complex $\Sigma$ is a convex ear-decomposition $\Sigma_{1}, \Sigma_{2}, \ldots, \Sigma_{m}$ in which $\Sigma_{1}$ is a PS- $(d-1)$-sphere while each $\Sigma_{i}$, for $i \geq 2$, is a PS- $(d-1)$-ball.

Proposition 5. If a pure rank-d simplicial complex admits a combinatorial eardecomposition, then it has a shelling $S$ such that $\Delta(S, \Sigma)$ is a pure rank-d $M$ shellable poset. 
Proof. We saw earlier (Example 2 in Section 2.4) that there is a shelling of a PSsphere such that the lower-bound poset of the shelling is an M-poset. Thus we can shell $\Sigma_{1}$ in this manner. Now let $\Sigma_{2}=\bar{X} \times \Omega_{2}$, where $\Omega_{2}$ is a PS- $(d-|X|-1)$-sphere. Now we take the appropriate shelling of $\Omega_{2}$, and augment each lower and upper bound with the set $X$ and "attach" it to the shelling of $\Sigma_{1}$. From the boundary condition of the ear-decomposition, this clearly gives a shelling of $\Sigma_{1} \cup \Sigma_{2}$, and the resulting lower-bound poset is a well-defined $|X|$-join of M-shellable lower-bound posets associated with shellings of $\Sigma_{1}$ and $\Omega_{2}$, and hence is M-shellable. It is easy to see that continuing this process eventually gives us a shelling of $\Sigma$ with the required properties.

Once again, we find that this decomposition, which can be interpreted as some sort of higher-order shellability property, has an equivalent recursive formulation which has the flavor of the notion of $k$-decomposability of Provan and Billera.

Definition. A simplicial complex $\Sigma$ is $P S$-decomposable if it is pure (of rank- $d$, say) and either $\Sigma$ is PS- $(d-1)$-sphere, or there exists a nonempty face $F$ of $\Sigma$ such that

1. $\Sigma \backslash F$ is pure rank- $d$ and PS-decomposable, and

2. $\operatorname{link}(\Sigma, F)$ is a pure rank- $(d-|F|)$ and PS-decomposable.

Theorem 2. A pure rank-d simplicial complex $\Sigma$ is PS-decomposable if and only if admits a PS ear-decomposition.

Proof. First, we will prove, by induction on the number of faces of $\Sigma$, that if it is a PS-decomposable simplicial complex then it admits a PS ear-decomposition. If $\Sigma$ has just one face, namely the empty set, then $\Sigma$ is the boundary of the 0 -simplex and hence the result is obviously true. More generally, the proof is trivial if $\Sigma$ is a PS$(d-1)$-sphere. If not, there exists a nonempty face $F$ satisfying the two conditions of the above definition. Now, let $\Psi_{1}, \Psi_{2}, \ldots, \Psi_{m}$ be a PS ear-decomposition of $\Sigma \backslash F$ and $\Omega_{1}, \Omega_{2}, \ldots, \Omega_{k}$ be a PS ear-decomposition of $\operatorname{link}(\Sigma, F)$-both of which exist by the induction hypothesis. Now we define a decomposition of $\Sigma$ into a sequence of subcomplexes $\Sigma_{1}, \Sigma_{2}, \ldots, \Sigma_{m+k}$, where $\Sigma_{i}=\Psi_{i}$ for $i=1,2, \ldots, m$ and $\Sigma_{m+j}=\bar{F} \times \Omega_{j}$ for $j=1,2, \ldots, k$. We claim that this is a PS ear-decomposition for $\Sigma$. It is easy to see that for each $i=2,3, \ldots, m+k, \Sigma_{i}$ is a PS- $(d-1)$-ball, while $\Sigma_{1}$ is a PS- $(d-1)$-sphere. Now, we observe that the intersection condition for this to be a PS ear-decomposition needs to be checked only for the PS-balls $\Sigma_{m+1}, \Sigma_{m+2}, \ldots, \Sigma_{m+k}$.

It is evident that for $i=2,3, \ldots, k$ we have

$$
\partial \Sigma_{m+i}=\left(\partial \bar{F} \times \Omega_{i}\right) \cup\left(\bar{F} \times \partial \Omega_{i}\right) .
$$

Now,

$$
\begin{aligned}
\Sigma_{m+i} & \cap\left(\bigcup_{l=1}^{m+i-1} \Sigma_{l}\right)=\left[\Sigma_{m+i} \cap(\Sigma \backslash F)\right] \cup\left[\Sigma_{m+i} \cap\left(\bigcup_{l=1}^{i-1} \Sigma_{m+l}\right)\right] \\
= & {\left[\left(\bar{F} \times \Omega_{i}\right) \cap(\Sigma \backslash F)\right] \cup\left[\bar{F} \times\left(\Omega_{i} \cap\left(\bigcup_{l=1}^{i-1} \Omega_{l}\right)\right)\right] } \\
= & {\left[\partial \bar{F} \times \Omega_{i}\right] \cup\left[\bar{F} \times \partial \Omega_{i}\right] } \\
= & \partial \Sigma_{m+i}
\end{aligned}
$$


Also, $\Sigma_{m+1} \cap(\Sigma \backslash F)=\left[\left(\bar{F} \times \Omega_{1}\right) \cap(\Sigma \backslash F)\right]=\partial \bar{F} \times \Omega_{1}=\partial \Sigma_{m+1}$. Thus we have shown that the ordered sequence $\Sigma_{1}, \Sigma_{2}, \ldots, \Sigma_{m+k}$ is indeed a PS eardecomposition of $\Sigma$.

Now assume that a pure rank- $d$ simplicial complex $\Sigma$ has a PS ear-decomposition; then we will prove by induction on the number $m$ of subcomplexes of the eardecomposition that $\Sigma$ is PS-decomposable. The result is obviously true for $m=$ 1. Now for $m>1$ suppose $\Sigma_{m}$, the last subcomplex of the combinatorial eardecomposition $\Sigma_{1}, \Sigma_{2}, \ldots, \Sigma_{m}$, is of the type $\Sigma_{m}=\bar{F} \times \Psi_{m}$, where $\Psi_{m}$ is a PS-sphere. Then it is evident that $\operatorname{link}(\Sigma, F)=\Psi_{m}$, which is obviously PSdecomposable. Also, $\Sigma \backslash F$ admits a PS ear-decomposition $\Sigma_{1}, \Sigma_{2}, \ldots, \Sigma_{m-1}$ and hence by induction is PS-decomposable. Hence we have shown that $\Sigma$ is PSdecomposable, as required.

Corollary 3. Let $\Sigma$ be a pure rank-d PS-decomposable simplicial complex. Then $\Sigma$ is shellable and the h-vector of $\Sigma$ is the set of rank-numbers of some pure rank-d $M$-shellable poset. In particular, the h-vector of $\Sigma$ satisfies equations (1), (2), and (3).

\section{Application to MATRoid COMPlEXeS}

We will apply the results of the last two sections to matroid complexes, and this results in a proof of Hibi's conjecture and another proof of the Brown-Colbourn alternating sum inequalities. In what follows, we will denote the matroid complex associated with a matroid $M$ by $\Psi(M)$. We will denote deletion and contraction by a subset $X$ of the ground set $E$ of $M$ by $M-X$ and $M / X$ respectively. We will denote the uniform matroid of rank- $d$ on $n$ elements by $U_{d, n}$.

Theorem 3. A coloop-free matroid complex $\Psi(M)$ is PS-decomposable.

Proof. We prove the result by induction on the number of elements of $\Psi(M)$. Since $M$ is coloop free, if $\Psi(M)$ has one element, then $M$ has rank 0 and $\Psi(M)$ is the boundary of the 0 -simplex, and the result follows. Now, we can assume that $d \geq 1$. For any (non-loop) element $e$ of $M$, let

$$
X=\{e\} \cup\{x \in E:\{x, e\} \text { is a (2-element) cocircuit of } M\} .
$$

It is easily seen using the (co)circuit exchange axioms for matroids that if $\{a, b\}$ and $\{a, c\}$ are both (2-element) cocircuits in $M$, then so is $\{b, c\}$. In particular, being in a 2-element cocircuit is an equivalence relation on the non-loop elements of $M$, and hence the vertices (rank-1 elements) of $\Psi(M)$ can be partitioned into series classes (with each loop being regarded as an individual series class). The set $X$ defined above is such a series class, and we observe that for any element $x \in X$, $X \backslash\{x\}$ is precisely the set of coloops of the matroid $M-\{x\}$. Another important property of the series class $X$ is that for any circuit $C$ of $M$, either $C \cap X=\emptyset$ or $C \cap X=X$. This follows from the fact that every pair of elements in $X$ is a cocircuit and hence cannot intersect the circuit $C$ in a single element. In particular, if the series class $X$ is dependent in $M$, then $X$ is, in fact, a circuit of $M$. Further, since $X \subseteq C$ is not possible, in this case, for any circuit $C$ of $M$, we must have $C \cap X=\emptyset$. We conclude, therefore, that if $X$ is a dependent series class, then $X$ is a circuit which is disconnected from the rest of the matroid.

Now for the matroid $M$, exactly one of the following two possibilities is true.

a. Every series class $X$ of the matroid $M$ is dependent in $M$. 
From the above discussion it follows that, in this case, $M$ is a direct sum of circuits. Hence $\Psi(M)$ is the direct product of boundaries of simplices, that is, it is a PS- $(d-1)$-sphere.

b. Some series class $X$ is independent in $M$.

If $X$ is an independent series class of $M$, then clearly $\operatorname{link}(\Psi(M), X)=\Psi(M / X)$, and since $M / X$ has no coloops, it is PS-decomposable by induction. Since $X$ is a series class, an independent set of $M$ belongs to $\Psi(M) \backslash X$ if and only if it is the disjoint union of an independent set of the matroid $M-X$ and some proper subset of $X$. In other words,

$$
\Psi(M) \backslash X=\Psi(M-X) \times \Psi\left(U_{|X|-1,|X|}\right) .
$$

Now $M-X$ has no coloops since $X$ is a series class of $M$, and also $U_{|X|-1,|X|}$ has no coloops. In particular, we have shown that $\Psi(M) \backslash X$ is a pure rank-d coloop-free matroid complex, and hence by induction, it is PS-decomposable.

Therefore, we have shown above that either (a) $\Psi(M)$ is a PS- $(d-1)$-sphere, or (b) there exists a face $X$ of $\Psi(M)$, such that $\Psi(M) \backslash X$ is a pure rank- $d$ PSdecomposable complex and $\operatorname{link}(\Psi(M), X)$ is also a PS-decomposable complex. It follows, hence, that $\Psi(M)$ is PS-decomposable.

Example 3. Let $M$ be the graphic matroid of the graph $K_{4}$ with the edgeset labelled as per the topmost graph of Figure 1. An implementation of the recursive decomposition used in the proof of the above theorem is demonstrated in this figure.

This decomposition gives rise to the following shelling of the matroid complex:

$$
\begin{aligned}
S=\{ & {[\emptyset, 134],[5,135],[45,145],[2,234],[25,235],[245,245],[12,123], } \\
& {[124,124],[6,126],[46,146],[36,236],[346,346],[56,356],[456,456], } \\
& {[156,156],[256,256]\} }
\end{aligned}
$$

The six subcomplexes of the PS ear-decomposition are shown in Figure 2. The M-shelling of $\Delta(S, M)$ of the poset of lower bounds of this shelling is shown in Figure 3 , with solid dark lines indicating the cover relations that appear in M-intervals. The lighter solid lines are the cover relations in $\Delta(S, M)$ which correspond to the join operations described in the proof of Proposition 5 . The dashed lines indicate the remaining cover relations of $\Delta(S, M)$.

We should point out that the poset of lower bounds has been studied by Dawson [13] and Purtill [4] for the shelling $S_{l e x}$ of the matroid complex which is given by the lexicographic order on the bases induced by an arbitrary but fixed total order on the ground set. For instance, they have shown that the associated poset, which we denote by $\Delta\left(S_{\text {lex }}, M\right)$, is an interval greedoid (see [4]). However, an example due to C. Athanasiadis (private communication) shows that $\Delta\left(S_{l e x}, M\right)$ is not even M-partitionable in general.

Let us define the H-polynomial of the matroid $M$ as the polynomial $H(M ; t)=$ $\sum_{i=0}^{d} h_{i} t^{d-i}$, where $d$ is the rank of the matroid and $\left(h_{0}, h_{1}, \cdots, h_{d}\right)$ is the $h$-vector of $M$. Thus $H(M ; t)=T(M ; t, 1)$, where $T(M ; x, y)$ is the Tutte polynomial of the matroid $M$ [4]. The following corollary can also be proved directly using the theory of the Tutte polynomial, and this approach is outlined in the communication [10], which is an announcement for the results of this paper. 


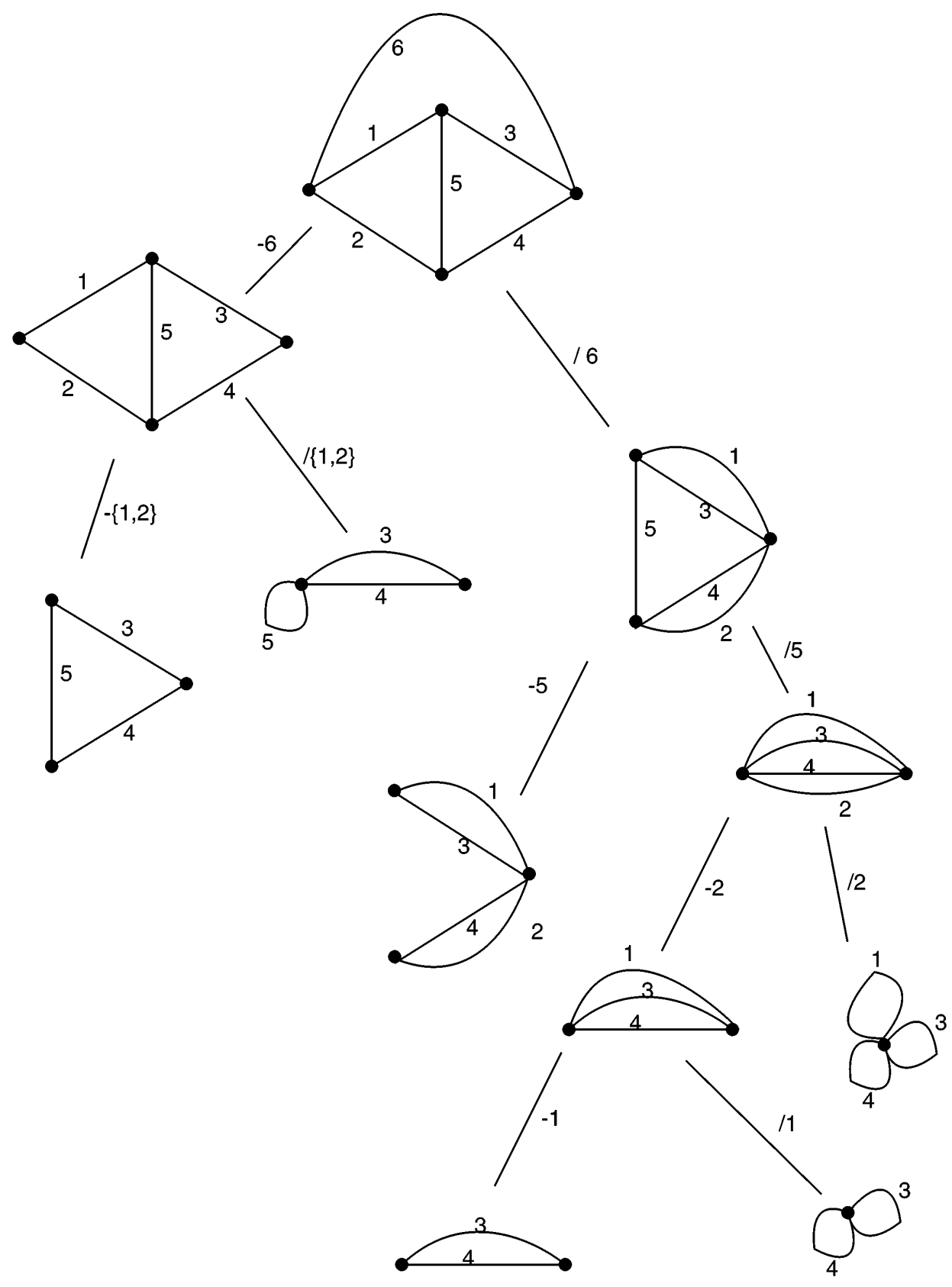

Figure 1

Corollary 4. For a coloop free matroid $M$ of rank $d$, we have the following:

1. If $M$ has $m$ connnected components, each of which is a circuit of size $d_{i}+1$, $i=1,2, \ldots, m$ then

$$
H(M ; t)=u\left(d_{1} ; t\right) u\left(d_{2}, t\right) \cdots u\left(d_{m} ; t\right)
$$



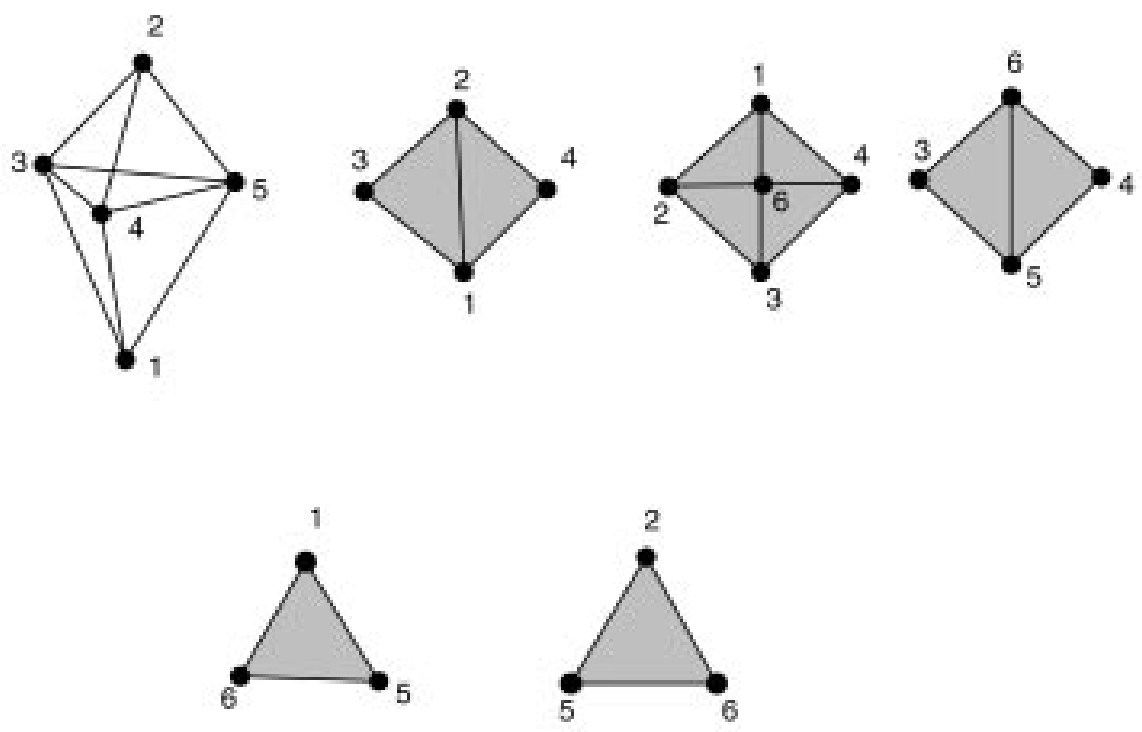

FiguRE 2

Otherwise, for any independent series class $X$ of $M$ with $|X|=k+1$,

$$
H(M ; t)=u(k ; t) H(M-X ; t)+H(M / X ; t) .
$$

2. The h-vector $\left(h_{0}, h_{1}, \cdots, h_{d}\right)$ of a coloop-free matroid $M$ satisfies the inequalities (1), (2), and (3).

For the graphic matroid of $K_{4}$ discussed in Example 3, the expansion for $H(M ; t)$ that is defined recursively in the previous corollary can be read off "top down" from the M-shelling of Figure 3 as follows:

$$
H(M ; t)=(1+t)\left(1+t+t^{2}\right)+(1+t)+(1+t)(1+t)+(1+t)+1+1 .
$$

It would be interesting to examine whether the existence of such an expansion can be used to derive new results about the location of roots of this polynomial

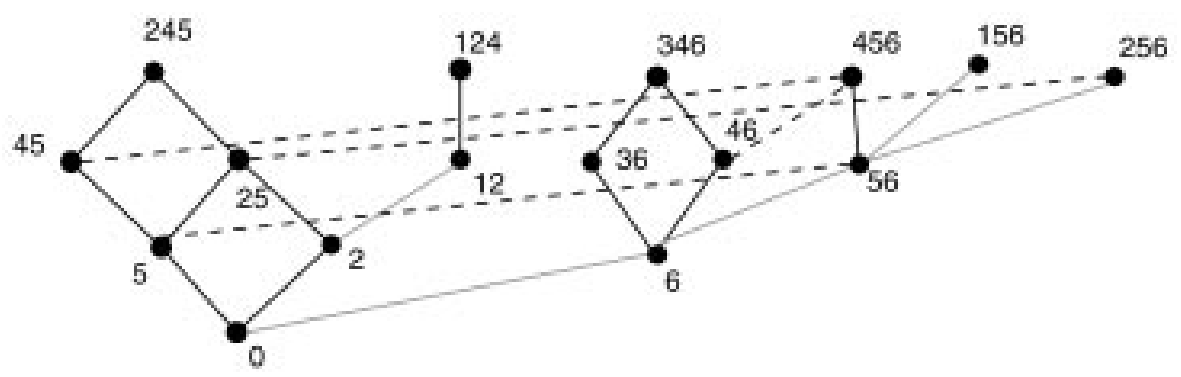

FiguRE 3 
and other related polynomials [7], [11]. The PS ear-decomposition may also be of interest in studying the matroid-basis graph, which is in some sense dual to the geometric realization of $\Psi(M)$, and hence inherits a corresponding decomposition. We leave the detailed analysis of these issues to future research.

It was brought to our attention by G. Ziegler (private communication) that PSspheres also appear (under a different name) in the work of Dayton and Weibel [15] on hyperplane arrangements. Ziegler [33] generalized some of their results to the combinatorial framework of the broken-circuit complex of a matroid (see [9], [4] for definitions). His work is essentially an extension of Björner's construction of homology cycles for matroid complexes discussed in the introduction, except that in this case, the original broken-circuit complex is not the union of the subcomplexes. Unfortunately, our techniques do not apply directly to broken-circuit complexes. In fact, the example of a broken circuit complex given by Brylawski in [9] has an $h$-vector $(1,2,3,1)$ and hence cannot admit a convex ear-decomposition. However, it is conceivable that some modification of our approach may indeed be succesful in developing an analogous theory for these complexes. Any result in this direction would be of significant interest, since it would have implications for the coefficients of chromatic polynomials of graphs, about which very little is known.

We will conclude with remarks about the consequences of this work to the following fundamental question in the study of matroid invariants.

Is there a numerical characterization for the set of h-vectors of matroids?

In this paper, we have shown that the $h$-vector of any coloop-free rank- $d$ matroid is the set of rank-numbers of some M-shellable poset. It is only natural to combine our results with Stanley's conjecture, and so we define a shellable O-sequence as the degree sequence of a pure, M-shellable order ideal of monomials.

Conjecture 3. The h-vector of a coloop-free matroid complex is a shellable $O$ sequence.

This conjecture naturally suggests the following problem.

Problem. Find a numerical characterization for shellable O-sequences.

It is interesting that the $f$-vectors of simplicial complexes and O-sequences have known numerical characterizations due to Kruskal and Katona and Macaulay [1] respectively, while the numerical characterizations for $f$-vectors of pure simplicial complexes or pure O-sequences are not known and are widely assumed to be intractable (see Ziegler's remarks on page 284 of [34]). However, we have seen that $f$-vectors of pure rank- $d$ shellable simplicial complexes do have such a characterization through their $h$-vectors, thanks to Stanley and Macaulay. In view of this background, it is reasonable to expect the problem of numerically characterizing shellable O-sequences to be tractable (or at least, more so than characterizing pure O-sequences). It would indeed be worthwhile to develop a theory of M-shellability of pure order ideals of monomials and define an appropriate invariant analogous to the $h$-vector of shellable complexes. It is well-known that the combinatorial property of shellability of simplicial complexes, in some sense, is a concrete manifestation of the more general property of being Cohen-Macaulay ([28], [26]). This property has two equivalent formulations: one is based on the homology of the simplicial complex, while the other is based on a purely ring-theoretic property of the Stanley-Reisner ring associated with the complex ([2], [28]). It is possible that M-shellability of an order ideal of monomials correponds to a more general algebraic theory in the ring-theoretic setting, which can be used to develop results analogous to Stanley's theory for $h$-vectors of Cohen-Macaulay complexes. 


\section{ACKNOWLEDGEMENTS}

Thanks to Scott Provan and Neil Stoltzfus for helpful discussions, and to Günter Ziegler and an anonymous referee for a careful reading of earlier versions of the manuscript.

\section{REFERENCES}

1. I. Anderson (1987). Combinatorics of Finite Sets, Oxford Science Publications, Oxford University Press. MR 89b:05001

2. L. J. Billera (1984). Polyhedral Combinatorics and Commutative Algebra, in Mathematical Programming: The State of the Art, (A. Bachem, B. Korte, Eds.), Springer-Verlag, 57-77. MR 84m:52009

3. A. Björner (1980). Shellable and Cohen-Macaulay partially ordered sets, Trans. Amer. Math. Soc. 260, 159-183. MR 81i:06001

4. A. Björner (1992). Homology and Shellability of Matroids and Geometric Lattices, in Matroid Applications, (N. White, Ed.), Encyclopaedia of Mathematics and its Applications, Cambridge University Press, 226-283. MR 94a:52030

5. A. Björner, M. Las Vergnas, B. Sturmfels, N. White, G. Ziegler (1992). Oriented Matroids, Cambridge University Press, Cambridge. MR 95e:52023

6. A. Björner (1996). Topological Methods, in Handbook of Combinatorics, Vol. II, (R. Graham, M. Grötschel and L. Lovaśz, Eds.), North-Holland, Amsterdam, pp. 1819-1872. MR 96m:52012

7. J.I. Brown and C.J. Colbourn (1992). Roots of the reliability polynomial, SIAM J. of Discrete Math. 5, 571-585. MR 93g:68005

8. H. Bruggesser and P. Mani (1971). Shellable decompositions of cells and spheres, Math. Scand. 29, 197-205. MR 48:7286

9. T.H. Brylawski (1977). The broken-circuit complex, Trans. Amer. Math. Soc. 234, 417-433. MR 80a:05055

10. M.K. Chari (1995). Matroid inequalities, Discrete Math. 147, 283-286. MR 96j:05031

11. C. J. Colbourn (1993). Some open problems on reliability polynomials, DIMACS Technical Report no. 93-28, Congr. Numer. 93 (1993), 187-202. MR 95c:05121

12. R. Cordovil (1985). On simplicial matroids and Sperner's Lemma, in Matroid Theory and its Applications, L. Lovasz \& A. Recski (Eds), Colloq. Math. Soc. Janos Bolyai, 40 North Holland, Amsterdam and Budapest, 97-105. MR 87g:05065

13. J. E. Dawson (1984). A collection of sets related to the Tutte polynomial of a matroid, in Lecture Notes in Mathematics. 1073, Springer Verlag, Berlin, 193-204. MR 85k:05032

14. G. Danaraj and V. Klee (1974). Shellings of spheres and polytopes, Duke Math. J. 41, 443-451. MR 49:9852

15. B.H. Dayton and C.A. Weibel (1980). K-theory of Hyperplanes, Trans. Amer. Math. Soc. 257, 119-141. MR 81e: 18015

16. T. Hibi (1989). What can be said about pure O-sequences?, Journal of Comb. Theory, Ser A. 50, 319-322. MR 90d:52012

17. T. Hibi (1992). Face numbers inequalities for matroid complexes and Cohen-Macaulay types of Stanley-Reisner rings of distributive lattices, Pacific Journal of Math. 154, 253-264. MR 93e: 52027

18. G. Kalai (1991). The Diameter of Graphs of Convex Polytopes and f-Vector Theory, DIMACS Series in Discrete Mathematics and Theoretical Computer Science 4, 387-411. MR 92h:52010

19. L. Lovasz and M.D. Plummer (1986). Matching Theory, Elsevier Science Pub. Co., New York. MR 88b:90087

20. J. R. Munkres (1984). Elements of Algebraic Topology, Addison-Wesley, Menlo Park. MR 85m:55001

21. P. McMullen (1970). The maximum number of faces of a convex polytope, Mathematika 17, 179-184. MR 44:921

22. P. McMullen (1971). The number of faces of simplicial polytopes, Israel J. Math. 9, 559-570. MR 43:3914

23. P. McMullen (1993). On Simple Polytopes, Inventiones Math. 113, 419-444. MR 94d:52015 
24. J.G. Oxley (1992). Matroid Theory, Oxford University Press. MR 94d:05033

25. J.S. Provan and L.J. Billera (1980). Decompositions of simplicial complexes related to diameters of convex polyhedra, Math. of O.R. 5, 579-594. MR 82c:52010

26. R.P. Stanley (1977). Cohen-Macaulay complexes, in Higher Combinatorics, M.Aigner (Ed.), Reidel, Dordrecht, 384-393. MR 58:28010

27. R. P Stanley (1980). The number of faces of simplicial convex polytopes, Adv. in Math. 35, 236-238. MR 81f:52014

28. R P. Stanley (1996). Combinatorics and Commutative Algebra, 2nd Edition, Progress in Mathematics, Vol. 41, Birkhäuser, Boston-Basel-Stuttgart. MR 85b:05002 (1st ed.)

29. R.P. Stanley (1986). Enumerative Combinatorics, Vol. I., Wadsworth-Brooks/Cole. MR 87j:05003

30. R.P. Stanley (1993). A monotonicity property of $h$-vectors and $h^{*}$-vectors, Europ. J. Combin. 14, 251-258. MR 94f:52016

31. R.P. Stanley (1989). Log-Concave and Unimodal Sequences in Algebra, Combinatorics and Geometry, Annals of the New York Academy of Sciences. 576, 500- 535. MR 92e:05124

32. D.J.A Welsh (1976). Matroid Theory, Academic Press, London. MR 55:148

33. G.M.Ziegler (1992), Matroid shellability, $\beta$-systems, and affine hyperplane arrangements. Journal of Algebraic Combinatorics 1, 283-300. MR 93j:52022

34. G.M. Ziegler (1995), Lectures on Polytopes, Graduate Texts in Mathematics 152, Springer Verlag, New York. MR 96a:52001

Department of Mathematics, Louisiana State University, Baton Rouge, Louisiana 70803

E-mail address: chari@math.lsu.edu 